\title{
Relação entre a prática da caminhada não supervisionada e fatores de risco para as doenças cardiovasculares em adultos e idosos
}

\section{Relationship between the practice of unsupervised walking and risk factors for cardiovascular disease in adults and elderly}

\author{
Atila A. Trapé1; Ana L. Sacardo², Adriele F. Cássia ${ }^{2}$, Henrique L. Monteiro ${ }^{3}$, Anderson S. Zago ${ }^{3}$
}

\begin{abstract}
RESUMO
Introdução: A adoção de um estilo de vida ativo através da prática da caminhada é altamente recomendada pelos profissionais de saúde com o intuito de evitar, minimizar ou reverter diversos agravos de saúde que podem comprometer a qualidade de vida da população. Objetivo: Verificar se a prática da caminhada não supervisionada possui estímulo suficiente para a redução de fatores de risco de doenças cardiovasculares em adultos e idosos. Métodos: Participaram deste estudo 225 homens e mulheres saudáveis que foram divididos em 4 grupos: sedentário (G0), caminhada (G1), caminhada e uma atividade extra (G2) e, caminhada e duas atividades extras (G3). Todos os participantes realizaram as seguintes avaliações: International Physical Activity Questionnaire (IPAQ) - versão curta, bateria de testes de aptidão física (AAHPERD), pressão arterial, perfil lipídico e composição corporal. Resultados: Os resultados apontaram que a prática da caminhada não supervisionada (G1) promoveu benefícios limitados à saúde quando comparado ao grupo G3 (índice de aptidão funcional geral $=273,4 \pm 111$ vs $340,6 \pm 92$; pressão arterial diastólica $=80,0 \pm 8$ vs $75,4 \pm 7 \mathrm{mmHg}$; HDL colesterol $=44,3 \pm 10$ vs $50,1 \pm 10$ $\mathrm{mg} / \mathrm{dL}$ respectivamente). Conclusão: Participar de maior número de atividades foi benéfico para diminuir os fatores de risco de doenças cardiovasculares quando comparados ao grupo sedentário e praticantes apenas de caminhadas.
\end{abstract}

Palavra-chave: Exercício Físico, Capacidade aeróbia, aptidão física, saúde, envelhecimento.

\section{Introdução}

Em praticamente todos os países, a proporção de pessoas acima dos 60 anos está aumentando de forma expressiva por dois fatores principais, a saber: o aumento da expectativa de vida e o declínio das taxas de natalidade. ${ }^{1}$ A Organização Mundial de Saúde $(\mathrm{OMS})^{2}$ aponta que o número indivíduos acima desta faixa etária dobrou desde 1980 e projeta-se para 2050 que o número de pessoas com mais de 80 anos au-
1. Professor do CEFER. Mestre em Saúde na Comunidade, Departamento de Medicina Social, Faculdade de Medicina / USP Ribeirão Preto

2. Professoras de Educação Física. Escola de Educação Física e Esporte de Ribeirão Preto / USP - Ribeirão Preto

3. Docentes do Departamento de Educação Física. Departamento de Educação Física, Faculdade de Ciências / UNESP - Bauru

Conflitos de interesse: os autores declaram não haver nenhum conflito de interesse
Correspondência:

Prof. Dr. Anderson Saranz Zago Faculdade de Ciências-UNESP. Depto de Educação Física, Av. Eng. Luiz Edmundo Carrijo Coube, $n^{\circ}$ 14-01, Vargem Limpa

CEP: 17033-360 / Bauru - SP aszago@fc.unesp.br

Artigo recebido em 19/08/2013 Aprovado para publicação em 05/02/2014 
mentará em quatro vezes atingindo a marca de 395 milhões de idosos em todo mundo.

Entretanto, a maior preocupação não está no número de idosos, mas na forma como as pessoas envelhecem. O processo de envelhecimento geralmente está acompanhado por inúmeros fatores deletérios decorrentes de alterações funcionais e estruturais que ocorrem com o avanço da idade, os quais comprometem significativamente a qualidade de vida desta população ${ }^{3}$. Dentre essas alterações, destaca-se a diminuição da aptidão física (força muscular, coordenação, capacidade aeróbia, flexibilidade e equilíbrio), que possui relação direta com a realização de atividades cotidianas $^{4,5} \mathrm{e}$, o aumento da vulnerabilidade em adquirir alguma doença, especialmente às doenças cardiovasculares (DCV), que possuem elevado número de fatores de risco que se acentuam com o processo natural de envelhecimento, como por exemplo, hipertensão arterial, diabetes, obesidade, dislipidemias, dentre outros. ${ }^{6,7,8}$

Com base nessas evidências, baixos indicadores de aptidão física associada a maior prevalência de fatores de risco de doenças cardiovasculares (FRDCV) pode comprometer a qualidade de vida do idoso, expondo-o a um elevado risco de morbidade e mortalidade. Entretanto, por se tratar de fatores de risco modificáveis, ambos podem ser revertidos ou atenuados através da mudança no estilo de vida, incluindo especialmente alterações na dieta e na prática regular de exercícios físicos. ${ }^{9-12}$

Desta forma, a adoção de um estilo de vida ativo mediado pela prática regular de exercícios físicos tem sido considerada como a principal atitude para evitar, minimizar ou reverter os declínios na aptidão física e nos FRDCV que frequentemente acometem os ido$\operatorname{sos}^{9-12}$. A esse respeito, a literatura aponta que estes benefícios estão relacionados à prática de exercícios físicos, desde que praticados com intensidade, frequência e durações adequadas para cada indivíduo ${ }^{9-12}$.

No entanto, à medida que as pessoas envelhecem, ocorre, concomitantemente, a redução da participação em programas de exercícios físicos ${ }^{11,13}$. Apesar dos indicadores positivos inerentes a uma vida ativa (controle das dislipidemias, melhora nos quadros de diabetes mellitus, obesidade, pressão sanguínea, dentre outros) o comportamento sedentário prevalece e tem se acentuado na população em geral. ${ }^{9,13,14}$ De acordo com o Colégio Americano de Medicina do Esporte $(\mathrm{ACSM})^{15}$ o mínimo recomendado para a prática de exercício físico deveria ser de 150 minutos de exercício físico moderada por semana ou 30 minutos de exercício físico na maioria dos dias da semana. No entanto, Hallal et al. ${ }^{16}$ com base em estudos epidemiológicos, afirmam que $31 \%$ da população mundial é atualmente considerada inativa. Este quadro se agrava pelo comprometimento precoce da autonomia e independência, devido à relação direta entre o decréscimo da prática de exercícios físicos e piores condições de saúde. ${ }^{15,17}$

Por outro lado, visando contrapor-se ao sedentarismo e à melhora da qualidade de vida do idoso, muitos profissionais da área de saúde recomendam à população a prática da caminhada que, por melhorar a capacidade aeróbia e promover benefícios significativos tanto para a aptidão física geral quanto para a redução dos FRDCV. ${ }^{18,19,20}$ No entanto, quando praticada sem a supervisão de um profissional, o que geralmente ocorre, pois é habitualmente realizada em praças e parques das cidades, há duvidas se este exercício físico atinge a intensidade, frequência e duração adequada para promover os benefícios esperados.

Desta forma, apesar de ser praticada e recomendada pelos profissionais da saúde, será que a realização da caminhada não supervisionada é suficiente para promover os benefícios fisiológicos esperados em termos de aumento da aptidão física e diminuição dos FRDCV ?

Outro aspecto a ser ressaltado é que, de acordo com a OMS e com as recomendações do ACSM, os idosos deveriam realizar, além de atividades que envolvam a melhora da capacidade aeróbia (como a caminhada), outros exercícios físicos que contemplem capacidades físicas, como força muscular, flexibilidade e equilíbrio.

No entanto, a prática da caminhada, na maioria das vezes, além de ser praticada sem supervisão, estaria abrangendo, predominantemente um único componente da aptidão física, a capacidade aeróbia, sendo considerada, portanto, como uma atividade unicomponente. Desta forma, será que atividades complementares e supervisionadas em associação à caminhada (atividades multicomponentes) não teriam melhores efeitos sobre os parâmetros da aptidão física e FRDCV em adultos e idosos?

Neste contexto, o objetivo deste estudo foi avaliar se a prática da caminhada não supervisionada seria suficiente para reduzir os FRDCV e melhorar o nível de aptidão física em adultos e idosos em relação a indivíduos não praticantes e praticantes de exercícios físicos com características multicomponentes. 


\section{Metodologia}

Este projeto foi aprovado pelo Comitê de Ética em Pesquisa da Faculdade de Ciências Farmacêuticas - USP/Ribeirão Preto (CEP/FCFRP n $\left.{ }^{\circ} 172 / 2010\right)$, que está em cumprimento aos princípios éticos contidos na Declaração de Helsinki.

Participaram do estudo 225 homens e mulheres saudáveis da cidade de Ribeirão Preto que, além de assinarem um termo de consentimento livre e esclarecido, atendiam os seguintes critérios de inclusão, obtidos através de uma entrevista estruturada: idade entre 50 e 75 anos; não fumante; limite máximo de pressão arterial sistólica (PAS) de repouso de $159 \mathrm{mmHg}$ e de pressão arterial diastólica (PAD) $99 \mathrm{mmHg}$ (SBH Estágio 1 de hipertensão); índice de massa corporal (IMC) $\leq 40 \mathrm{~kg} / \mathrm{m}^{2}$; não ter nenhuma doença cardiovascular diagnosticada ou alguma restrição médica, particularmente as ortopédicas, que pudesse impedir a realização de exercícios físicos.

O número de participantes foi calculado com base nos testes estatísticos, considerando o nível de significância de $5 \%$ e poder do teste de $80 \%$. O maior tamanho amostral necessário para realização da Análise de Variância (ANOVA) consistiu em 194 indivíduos, entretanto foram acrescentados $10 \%$ ao valor do tamanho amostral calculado devido às eventuais perdas. A amostragem foi por conveniência e ocorreu em dois estágios, sendo que no primeiro foram selecionados idosos participantes de associações de aposentados e programas comunitários vinculados às Universidades e à Prefeitura da cidade de Ribeirão Preto/SP. Estes espaços públicos agregam a população idosa e são heterogêneas quanto a aspectos como condição socioeconômica e nível habitual de atividade física. No estágio II, todos os indivíduos de cada associação ou programa foram convidados a participar do estudo, com chances iguais de participação.

Os participantes inicialmente foram subdivididos de acordo com a quantidade de exercícios físicos regulares praticados, relatados através de uma anamnese e um questionário semi-estruturado que solicitava informações sobre quais exercícios físicos eram realizados, se era supervisionado por um profissional, como eram executados esses exercícios, a quanto tempo, dentre outras questões. Com base nessas informações foram formados quatro grupos: G0 - grupo sedentário, G1 - praticantes de caminhada, G2 - praticantes de caminhada e outro exercício físico (musculação ou hidroginástica ou dança ou alongamentos etc) e, G3 - praticantes de caminhada e outros dois tipos de exercícios físicos. Para ser incluído nos grupos ativos (G1, G2 ou G3) os participantes deveriam realizar os exercícios físicos supracitados num total de 150 minutos por semana, de acordo com as recomendações do $\mathrm{ACSM}^{15}$ preferencialmente subdivididos de acordo com os seguintes critérios: 3 a 4 vezes por semana com duração de 30 a 40 minutos por sessão a pelo menos quatro meses. Cabe ressaltar que a prática da caminhada dos três grupos ativos era realizada de forma não supervisionada por todos os participantes em parques ou praças próximas à residência e, os exercícios extras eram realizados em grupos de terceira idade, clubes ou academias sob supervisão de um profissional de educação física.

Para evitar os efeitos agudos do exercício físico sobre os resultados, os participantes foram orientados a não praticarem qualquer tipo de exercício físico nas 24 horas antecedentes as seguintes avaliações:

1. Nível habitual de atividade física (NHAF): Questionário Internacional de Atividade Física (IPAQ). Trata-se de um instrumento validado no Brasil pelo Centro de Estudos do Laboratório de Aptidão Física de São Caetano do Sul. ${ }^{21}$ Neste trabalho, utilizou-se da versão curta do questionário, que inclui os quatro componentes da atividade física: trabalho, meio de transporte, casa (trabalho, tarefas domésticas e cuidados com a família) e lazer (exercícios físicos, esporte e recreação). A classificação do nível habitual de atividade física foi obtida somando-se a frequência e duração de todas as atividades, categorizando posteriormente os participantes segundo o nível de atividade física habitual em: sedentários, insuficientemente ativos (A) e (B), ativos e muito ativos, conforme critérios descritos previamente $^{21}$;

2. Aptidão física: (a) Bateria de Testes da American Alliance for Health, Physical Education, Recreation and Dance (AAHPERD) - compreende a avaliação dos seguintes componentes de aptidão física: coordenação, flexibilidade, força muscular, agilidade e equilíbrio dinâmico e capacidade aeróbia, conforme descritos previamente. ${ }^{22}$ Os resultados obtidos foram classificados de acordo com os valores normativos da aptidão funcional, que possibilitou a obtenção do Índice de Aptidão Funcional Geral (IAFG) através da somatória do escorepercentil de cada teste, conforme descrito previamente $^{22}$ e; (b) Consumo máximo de oxigênio ( $\dot{\mathrm{VO}}_{2} \max$ ) indireto através do teste de 1 milha, que basicamente consiste em caminhar 1.600 metros no menor tempo possível. ${ }^{23}$ 
3. Avaliação da Pressão Arterial: Para a determinação dos valores de pressão arterial (PA) foram realizados três registros da PA em dias diferentes, utilizando-se um esfigmomanômetro aneróide com tamanho adequado para o diâmetro do braço dominante do participante e estetoscópio da marca Premium (manômetro de 0-300 mmHg e braçadeira para adulto), pelo mesmo avaliador, após o participante permanecer em repouso por cinco minutos na posição sentada. A média das três aferições da PA foi considerada como resultado final. Todo o procedimento de aferição da PA estava de acordo com as VI Diretrizes Brasileiras de Hipertensão Arterial. $^{24}$

4. Perfil lipídico e composição corporal: Após jejum de 12 horas, foram coletados $10 \mathrm{ml}$ de sangue venoso através da veia antecubital. Todo o procedimento foi realizado por profissional de enfermagem com experiência neste tipo de coleta. O perfil lipídico compreendeu as análises de colesterol total (CT), HDL-colesterol (HDL-c), LDL-colesterol (LDLc) e triglicérides (TG). Os reagentes utilizados para análise foram do mesmo lote (LABORLAB) e os métodos aplicados foram o método enzimático para CT e TG e, colorimétrico para o HDL-c. O LDL-c foi calculado por meio da equação de Friedewald. ${ }^{25}$ $\mathrm{O}$ índice de massa corporal $\left(\mathrm{IMC}=\right.$ peso/estatura $^{2}$ foi utilizado como indicador antropométrico através da mensuração do peso e estatura por meio de uma balança Filizola (modelo 31) e estadiômetro com resolução de $0,1 \mathrm{~kg}$ e $0,01 \mathrm{~m}$ respectivamente.

\section{Análise Estatística}

Foi realizada uma análise descritiva e, após verificar a normalidade dos dados, análise de variância tendo como variável independente a quantidade de prática realizada com teste de pos-hoc de scheffer. Para verificar a associação entre o número de atividades físicas praticadas e a chance de ocorrência de doenças cardiovasculares e metabólicas foi elaborado um modelo de regressão logística binária (qui-quadrado e Odds Ratio). O nível de significância foi determinado em $5 \%$.

\section{Resultados}

Após a divisão dos participantes em grupos de acordo com o número de atividades praticadas, a tabela 1 apresenta os resultados em quantidade/porcentagem de indivíduos em cada grupo com relação às variáveis idade, sexo e NHAF obtido pelo questionário IPAQ que se refere à percepção do idoso quanto à quantidade de atividade física realizada.

Tabela 1: Análise descritiva da idade, sexo e nível habitual de atividade física dos participantes, subdivididos em grupos de acordo com o número de atividades físicas praticadas.

\begin{tabular}{lrrrr}
\hline & G0 (n=66) & G1 (n=78) & G2 (n=50) & G3(n=31) \\
\hline $\begin{array}{l}\text { Idade (anos) } \\
\text { Sexo }\end{array}$ & $61,2 \pm 9,1$ & $60,5 \pm 10,5$ & $59,2 \pm 9,0$ & $62,6 \pm 8,1$ \\
$\quad$ & & & \\
$\quad$ Mulheres & $54(81,8 \%)$ & $58(74,4 \%)$ & $36(72,0 \%)$ & $20(64,5 \%)$ \\
$\quad$ Homens & $12(18,2 \%)$ & $20(25,6 \%)$ & $14(28,0 \%)$ & $11(35,5 \%)$ \\
Nível habitual de atividade física & & & & \\
$\quad$ Muito Ativo & $4(6,1 \%)$ & $8(10,3 \%)$ & $5(10,0 \%)$ & $4(12,9 \%)$ \\
Ativo & $15(22,7 \%)$ & $24(30,8 \%)$ & $22(44,0 \%)$ & $13(41,9 \%)$ \\
Irregularmente Ativo A & $13(19,7 \%)$ & $26(33,3 \%)$ & $12(24,0 \%)$ & $10(32,3 \%)$ \\
Irregularmente Ativo B & $22(33,3 \%)$ & $16(20,5 \%)$ & $10(20,0 \%)$ & $3(9,7 \%)$ \\
Sedentário & $12(18,2 \%)$ & $4(5,1 \%)$ & $1(2,0 \%)$ & $1(3,2 \%)$ \\
\hline
\end{tabular}

G0 - grupo sedentário, G1 - praticantes de caminhada, G2 - praticantes de 2 atividades físicas e, G3 - praticantes de 3 atividades físicas. O nível habitual de atividade física foi classificado de acordo com os critérios descritos previamente por Matsudo e colaboradores (2001). 
A figura 1 apresenta os resultados do e do IAFG dos participantes. Os três grupos ativos (G1, G2 e G3) apresentaram diferenças estatísticas quando comparados ao grupo não ativo (G0) em ambas as variáveis. Entretanto somente o IAFG discriminou os resultados entre os grupos ativos (G1 e G3).

Com relação à pressão arterial, nenhuma diferença foi apontada na PAS entre os quatros grupos, entretanto a PAD apontou diferenças dos grupos G2 e G3 comparativamente ao grupo G0 (Figura 2).
A análise do perfil lipídico não apontou diferenças significativas entre os quatro grupos nas variáveis CT e LDL-c. Diferença estatística significante do grupo G0 em relação aos grupos G2 e G3 foi observada para a variável TG e entre os grupos G0 e G3 para o HDL-c (Figura 3).

Em relação aos valores de composição corporal, todos os grupos ativos apresentaram diferenças na variável IMC quando comparados ao grupo não ativo (G0) (figura 4).
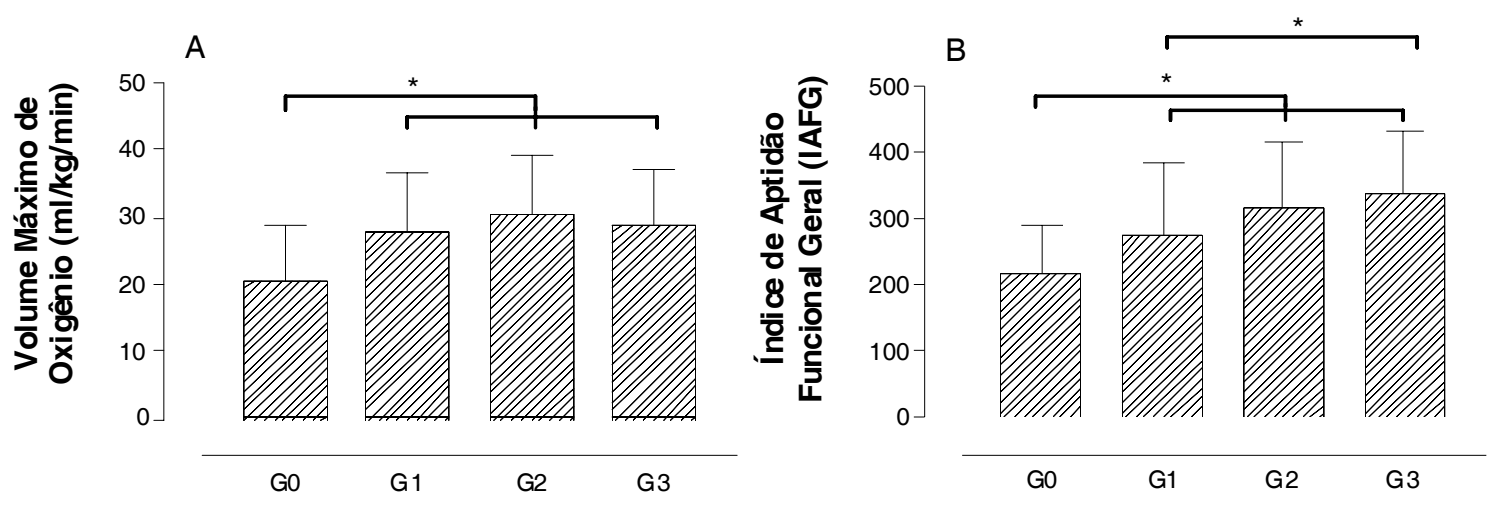

Figura 1: Consumo máximo de oxigênio (painel A) e índice de aptidão funcional geral (painel B) em adultos e idosos subdivididos de acordo com o número de atividades físicas praticadas (G0 - sedentário / G1 - praticantes de caminhada / G2 - praticantes caminhada e outra atividade / G3 - praticantes de caminhada e 2 outras atividades). ${ }^{*} \mathrm{p}<0.05$
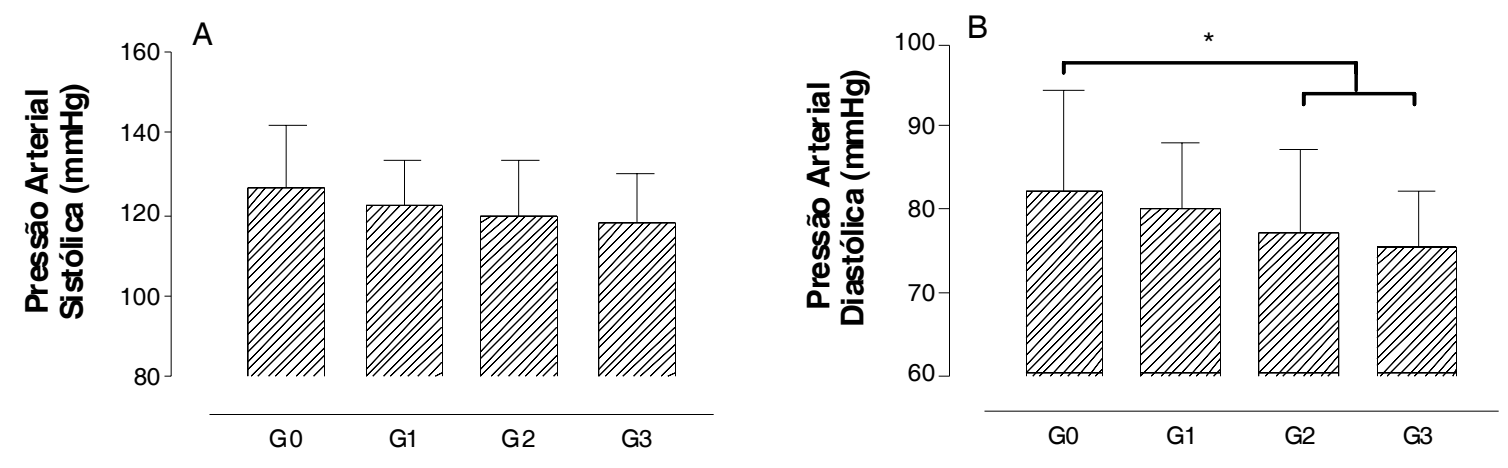

Figura 2: Pressão arterial sistólica (painel A) e diastólica (painel B) em adultos e idosos subdivididos de acordo com o número de atividades físicas praticadas (G0 - sedentário / G1 - praticantes de caminhada / G2 - praticantes caminhada e outra atividade / G3 praticantes de caminhada e 2 outras atividades). ${ }^{*} p<0.05$. 

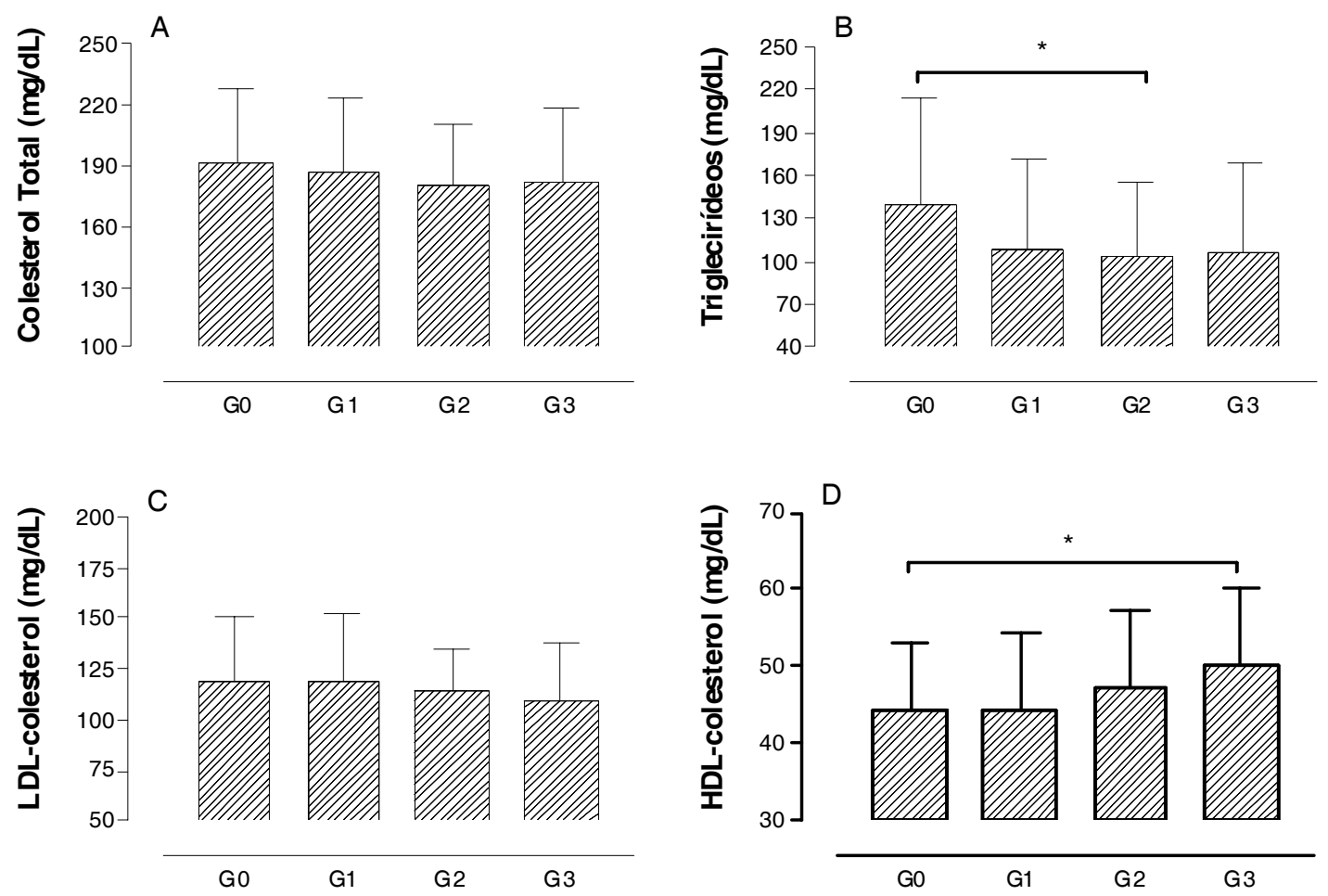

Figura 3: Valores de colesterol total (painel A), triglicerídeos (painel B), LDL-colesterol (painel C) e HDL-colesterol (painel D) em adultos e idosos subdivididos de acordo com o número de atividades físicas praticadas (G0 - sedentário / G1 - praticantes de caminhada / G2 - praticantes caminhada e outra atividade / G3 - praticantes de caminhada e 2 outras atividades). ${ }^{*} p<0.05$

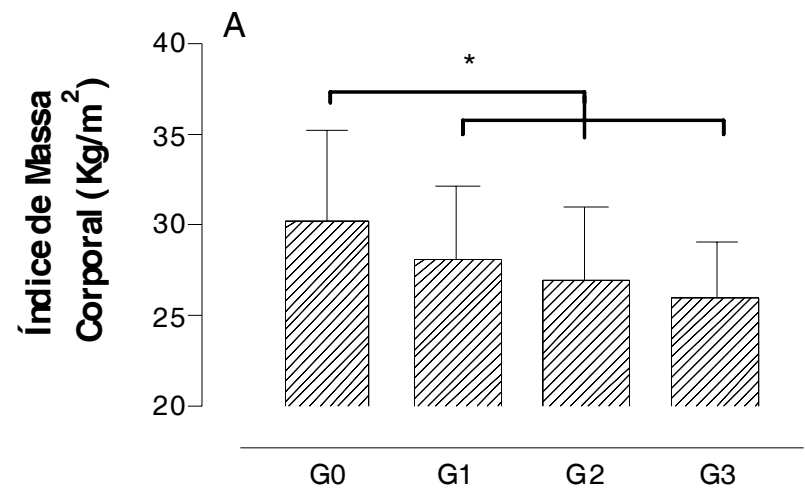

Figura 4: Índice de massa corporal em adultos e idosos subdivididos de acordo com o número de atividades físicas praticadas (G0 - sedentário / G1 - praticantes de caminhada / G2 - praticantes caminhada e outra atividade / G3 - praticantes de caminhada e 2 outras atividades). * $p<0.05$

A tabela 2 apresenta os resultados da análise de regressão logística binária indicando que o grupo G3, quando comparado ao grupo G0, apresentou menor risco de apresentar medidas de pressão arterial superiores aos valores de referência para normalidade $\left(\mathrm{OR}=0,33\left[_{\mathrm{O}} \mathrm{OR}_{\mathrm{IC} \%}: 0,11-0,98\right]\right)$, bem como de registrarem taxas de HDL-c alteradas $(\mathrm{OR}=0,15$ $\left.\left[\mathrm{OR}_{\mathrm{IC} 95 \%}: 0,05-0,43\right]\right)$. 
Tabela 2. Associação entre número de atividades realizadas e fatores de risco para doenças cardiovasculares de adultos e idosos.

\begin{tabular}{|c|c|c|c|}
\hline \multirow[t]{2}{*}{ Atividade Física } & \multicolumn{2}{|c|}{ Univariado } & \multirow{2}{*}{$\begin{array}{l}\text { Multivariado } \\
\mathrm{OR}^{\#}(\mathrm{IC} 95 \%)\end{array}$} \\
\hline & $\mathrm{CT}(\geq 200 \mathrm{mg} / \mathrm{dL})$ & Qui-quadrado & \\
\hline G0 & $42,4 \%$ & $P=0,199$ & - \\
\hline Gl & $35,9 \%$ & & - \\
\hline $\mathrm{G} 2$ & $26 \%$ & & - \\
\hline \multirow[t]{2}{*}{$\mathrm{G}$} & $35,5 \%$ & & - \\
\hline & $\mathrm{TG}(\geq 150 \mathrm{mg} / \mathrm{dL})$ & Qui-quadrado & $\mathrm{OR}^{\#}(\mathrm{IC} 95 \%)$ \\
\hline G0 & $33,3 \%$ & $P=0,164$ & - \\
\hline Gl & $19,2 \%$ & & - \\
\hline G2 & $20 \%$ & & - \\
\hline \multirow[t]{2}{*}{$\mathrm{G} 3$} & $22,6 \%$ & & - \\
\hline & $\mathrm{LDL}(\geq 160 \mathrm{mg} / \mathrm{dL})$ & Qui-quadrado & $\mathrm{OR}^{\#}(\mathrm{IC} 95 \%)$ \\
\hline G0 & $9,1 \%$ & $P=0,373$ & - \\
\hline Gl & $10,3 \%$ & & - \\
\hline G2 & $4 \%$ & & - \\
\hline \multirow[t]{2}{*}{$\mathrm{G}$} & $6,5 \%$ & & - \\
\hline & $\mathrm{HDL}(\leq 40 \mathrm{mg} / \mathrm{dL})$ & Qui-quadrado & $\mathrm{OR}^{\#}(\mathrm{IC} 95 \%)$ \\
\hline G0 & $72,7 \%$ & $P=0,001$ & 1.00 \\
\hline Gl & $62,8 \%$ & & $0.82(0,42-1,98)$ \\
\hline G2 & $52 \%$ & & $0.64(0,27-1,50)$ \\
\hline \multirow[t]{2}{*}{$\mathrm{G}$} & $22,6 \%$ & & $0.15(0,05-0,43)$ \\
\hline & $\mathrm{HA}(\geq 130 / 85 \mathrm{mmHg})$ & Qui-quadrado & $\mathrm{OR}^{\#}(\mathrm{IC} 95 \%)$ \\
\hline G0 & $45,5 \%$ & $P=0,004$ & 1.00 \\
\hline $\mathrm{Gl}$ & $35,9 \%$ & & $0.75(0,36-1,57)$ \\
\hline $\mathrm{G} 2$ & $26 \%$ & & $0.54(0,22-1,31)$ \\
\hline $\mathrm{G}$ & $19,4 \%$ & & $0,33(0,11-0,98)$ \\
\hline
\end{tabular}

G0 - grupo sedentário, G1 - praticantes de caminhada, G2 - praticantes de 2 atividades físicas e, G3 - praticantes de 3 atividades físicas.

OR= odds ratio; $\mathrm{IC} 95 \%=$ intervalo de confiança de 95\%; TG= triglicérides; $\mathrm{CT}=$ colesterol total; $\mathrm{LDL}=$ lipoproteína de baixa densidade; HDL= lipoproteína de alta densidade; ${ }^{\#}=$ ajustado por sexo, idade, etnia, hábito de fumar e risco coronariano. As variáveis com valores de $\mathrm{p}>0,20$ não foram incluídos no modelo. 


\section{Discussão}

Diversos estudos abordam os benefícios da adoção de um estilo de vida saudável incluindo a prática regular de exercícios físicos. Por orientação médica, ou incentivada por veículos de comunicação de massa, a prática da caminhada é fortemente recomendada, especialmente no que se refere à diminuição da prevalência dos fatores de risco para as doenças cardiovasculares e aumento da aptidão física, trazendo benefícios significativos para a qualidade de vida da população idosa ${ }^{13}$. No entanto, os resultados deste estudo apontam para benefícios limitados com a prática da caminhada quando realizada de forma isolada e não supervisionada. Por outro lado, quando associada a outros tipos de exercícios físicos, os resultados deste estudo apontam para benefícios significativos na aptidão física, pressão arterial, perfil lipídico e composição corporal.

Estes resultados ocorrem devido a dois fatores principais, a saber: a) falta de orientação de como a caminhada deve ser realizada; e, b) por trabalhar predominantemente um único componente da aptidão física (capacidade aeróbia) provocando benefícios limitados ao organismo.

Com relação ao primeiro fator, inicialmente este estudo avaliou o nível habitual de atividade física. Este resultado aponta que mesmo as pessoas integrantes do grupo G0 (sedentárias) alcançaram desempenho, em termos percentuais, muito próximos aos outros grupos ativos. Este dado indica que o IPAQ, da forma como está estruturado, não discrimina o nível de atividade física desta população.

O IPAQ, além de ser um questionário recomendado pela Organização Mundial de Saúde para estudos de base populacional, também é considerado como bom preditor de saúde auto-referida. ${ }^{26}$ No entanto, nossos resultados não permitem concluir que existe associação entre melhores escores no IPAQ com índices mais elevados da aptidão física. As justificativas para este desfecho podem estar relacionadas tanto a questão de terminologias quanto a prática inadequada de exercícios físicos. O primeiro aspecto leva em consideração a diferença entre os conceitos de "atividade física" e "exercício físico". Atividade física pode ser definida como qualquer movimento realizado pela musculatura esquelética que resulte em gasto energético superior aos valores de repouso, enquanto que, exercício físico é considerado como uma subcategoria de atividade física, porém deve ser previamente planejado, repetitivo e prescrito com objetivos bem definidos. ${ }^{27}$

Sobre esta ótica, por ser o IPAQ um instrumento de percepção subjetiva do nível de atividade física, os participantes podem ter informado serem fisicamente ativos, entretanto, esta resposta não permite concluir que, de fato, eles eram adeptos a prática regular de exercícios físicos. Por esta razão, apesar de muitos profissionais da área de saúde aconselharem a prática da caminhada como exercício físico, o fato de ser realizada sem supervisão e, portanto, sem controle de intensidade, duração e frequência, é possível que esta prática esteja mais voltada para a realização de atividades físicas do que de exercício físico propriamente dito.

No segundo aspecto, apesar de ser comum observar pessoas realizando a prática da caminhada em parques e praças, muitas o fazem sem orientação e, portanto, sem a intensidade necessária para prevenir a ocorrência de doenças crônicas não transmissíveis, como é o caso dos participantes desta pesquisa. Desta forma, os resultados deste estudo indicam que apesar dos participantes se considerarem ativos, especialmente os do grupo G1, não há evidências que esta prática esteja gerando reais benefícios para a saúde do praticante de caminhada.

Em relação ao segundo fator, a prática de apenas um componente da aptidão física certamente estaria limitando a gama de benefícios que a prática multicomponente propiciaria ao praticante. Neste caso, os resultados apresentados na figura 1 do presente estudo sustentam esta afirmação, pois independente de ser somente a caminhada ou a caminhada em associação a outra atividade, os resultados do são significativos e similares entre os grupos ativos. Dentre os diversos efeitos benéficos gerados ao organismo humano pela prática do exercício físico aeróbio, pode-se citar o aumento das enzimas oxidativas, aumento do número e tamanho das mitocôndrias, aumento da capilarização muscular e melhor transporte e extração muscular de oxigênio, contribuindo assim para um maior consumo máximo de oxigênio e, por consequência, melhor desempenho motor. ${ }^{28}$ Nesta direção, especificamente para a capacidade aeróbia, tanto a prática unicomponente (caminhada) quanto a multicomponente estariam promovendo tais benefícios. Entretanto, ao analisar o IAFG, um índice relacionado à aptidão física geral para realização de atividades cotidianas, observa-se valores mais elevados e de forma proporcional ao aumento do número de atividades pratica- 
das. Somente não foram encontradas diferenças entre os grupos G2 e G3 indicando que a associação de pelo menos um exercício físico com a caminhada deve ser recomendada.

Contudo, qual a interferência desses resultados nos FRDCV, especificamente na pressão arterial, perfil lipídico e composição corporal ?

Com relação à pressão arterial, há estudos que apontam para os benefícios do exercício físico sobre os níveis pressóricos de hipertensos, com significativo efeito hipotensor pós esforço. ${ }^{10,14,29}$ Entretanto os resultados do presente estudo indicam que somente a prática da caminhada não supervisionada não estaria repercutindo em melhoras nos níveis pressóricos de adultos e idosos. Somente os grupos que realizaram a prática multicomponente apresentaram menores valores na PA, especialmente na PAD. Não obstante, sobre estes resultados, cabe a ressalva de que o presente estudo é do tipo transversal enquanto que os citados anteriormente são longitudinais, motivo pelo qual não é possível concluir por relação entre exposição e efeito, sendo esta uma limitação da presente investigação. No entanto pode-se observar que maiores valores de pressão arterial foram encontrados nos grupos sedentários (G0) e praticantes apenas da caminhada (G1). Desta forma, em adição aos resultados encontrados na literatura, este estudo aponta que melhores resultados são encontrados com a prática multicomponente de exercícios físicos em detrimento da prática apenas da caminhada.

Em relação ao perfil lipídico, sabe-se que indivíduos fisicamente ativos apresentam maiores chances de apresentarem valores mais elevados de HDLc e menores de TG, LDL-c e VLDL colesterol quando comparados aos sedentários. ${ }^{30,31}$ Os resultados desse estudo indicaram diferenças significantes apenas para as TG e HDL-c. Esta diferença somente foi encontrada nos grupos que realizavam outro tipo de exercício físico associado à caminhada, para o TG e, duas atividades para o HDL-c.

Apesar dos estudos indicarem benefícios da prática de exercícios físicos no perfil lipídico ${ }^{32}$ o grupo G1 (praticante de caminhada não supervisionada) não apresentou mudanças significativas nestas variáveis. A esse respeito, sugere-se que a prática multicomponente se destaca quando se busca benefícios significativos para a saúde. Esta afirmação está de acordo com dados obtidos por Marques et al. ${ }^{33}$ ao observarem que o treinamento multicomponente foi mais efetivo do que o treinamento de resistên- cia para as mudanças no perfil lipídico e nas lipoproteínas plasmáticas.

Para a determinação do estado nutricional, o IMC é amplamente utilizado em estudos populacionais ${ }^{13}$ e sua associação com a atividade física/exercício físico é bastante explorada na literatura técnica pertinente. A esse respeito, Straznicky et al ${ }^{34}$ encontraram redução significativa dos valores de IMC no grupo com prática regular de exercícios aeróbios. Resultados similares também foram encontrados no presente estudo, pois tanto a caminhada não supervisionada (G1) quanto o treinamento multicomponente (G2 e G3) apresentaram diferenças quando comparado ao grupo sedentário (G0). No entanto, os resultados do presente estudo apontaram que os participantes que realizavam atividades multicomponentes, apresentaram uma tendência, mesmo que não significativa, de menores valores de IMC, fato que poderia estar indicando que a prática multicomponente possui a capacidade de otimizar os benefícios esperados com a prática de exercícios.

Em termos gerias, apesar de estes resultados fornecerem um indicativo de que somente a caminhada não supervisionada não resultaria em estímulo suficiente para promover saúde, é necessário considerar o efeito de outras variáveis que poderiam provocar efeito de confusão sobre os resultados obtidos. Desta forma, foi realizada uma análise de regressão logística binária, ajustando os dados às variáveis que poderiam interferir nos resultados, verificando a real influência do número de atividades realizadas com os FRDCV. A partir desta análise, o grupo G3 quando comparado ao grupo sedentário (G0), apresentou fator de proteção para as variáveis de PA e HDL-c.

Mello et al $^{35}$ realizaram estudo de intervenção com amostra randomizada, constituído por usuárias de Programa de Saúde da Família e observaram que mulheres praticantes de caminhada orientada, três vezes na semana (50 minutos por sessão), tiveram aumento significativo do HDL-c e redução significativa de TG, enquanto que, no grupo controle, o CT, HDLc, LDL-c e glicêmica de jejum aumentaram significativamente. Apesar das diferenças entre os momentos inicial e final, na comparação intergrupos não foram observadas diferenças estatísticas, com exceção da variável TG. A exemplo deste estudo, há outras pesquisas como a de Puglisi et al ${ }^{36}$ que analisaram o efeito da caminhada sobre a resposta da pressão arterial e perfil lipídico, no entanto, em nenhum dos casos foram utilizados modelos de regressão logística para 
avaliar fatores de risco ou de proteção. Por esta razão, por não ajustarem variáveis que possam determinar viés de interpretação, como idade e sexo, eventuais resultados positivos ou negativos podem ter sido determinados pela influência destas variáveis.

Por sua vez, Murtagh et $\mathrm{al}^{20}$, afirmam que... "médicos e profissionais de saúde pública estão em posição privilegiada para recomendar que seus pacientes aumentem os níveis de caminhada diária. Há evidências suficientes na literatura, para suportar que mesmo um pequeno aumento da caminhada diária é melhor que não caminhar, no entanto, ressaltam que é somente com aumento significativo da intensidade, volume e frequência que se pode obter benefícios cardiovasculares significativos".

De fato, Tambalis et $\mathrm{al}^{37}$, em estudo de revisão sistemática, reforçam a assertiva anterior, ao sustentarem que comparações entre diferentes intensidades de programas de exercícios aeróbios resultam em benefícios expressivos à saúde somente quando são realizados em intensidades adequadas.

É importante ressaltar que não é o objetivo desta pesquisa desestimular a recomendação para a prática da caminhada, mas alertar que, quando realizada de forma isolada e não supervisionada, esta prática pode promover benefícios limitados ao organismo, bem como acentuar o risco de complicações ortopédicas, metabólicas e cardiovasculares. Por outro lado, a associação da caminhada com outras formas de exercícios físicos pode maximizar os efeitos benéficos da prática regular do exercício físico em geral, culminando em efeitos reais para a saúde do praticante.
Conforme apontado anteriormente, estudos de corte transversais não permitem o esclarecimento da relação exposição e efeito, fato este que pode limitar as conclusões deste estudo, no entanto permite a associação entre as variáveis. Ainda, a presente investigação também apresenta outras limitações como a ausência de informações sobre: a condição sócio-econômica dos participantes; falta de registro sobre o tempo em que permaneciam sentados durante o dia; ausência do controle ou registro da dieta alimentar; e, ausência de outras avaliações que poderiam acrescentar informações para a discussão, como por exemplo, glicemia e circunferência da cintura, importantes indicadores de risco para doenças metabólicas e cardiovasculares respectivamente. Os autores sugerem que em futuras investigações sobre o tema, tais variáveis sejam acrescidas à investigação.

\section{Conclusão}

Este estudo indicou que a orientação para a prática da caminhada associada a outro tipo de exercício físico (atividade multicomponente) proporcionou melhores resultados nos FRDCV e no nível de aptidão física de adultos e idosos, sendo, portanto, uma recomendação que deveria ser incorporada pelos profissionais da área de saúde quando se objetiva melhorar a qualidade de vida e a saúde deste segmento populacional.

Agradecimentos: FAPESP - 09/54586-0

\section{ABSTRACT}

Remain physically active through the practice of walking is highly recommended by health professionals in order to avoid, minimize or reverse various health disorders that can compromise a good quality of life for the population. Objective: To verify if the non supervised walk has enough stimulation to reduce risk factors of cardiovascular disease in adults and the elderly. Methods: This study included 225 healthy men and women who were divided in 4 groups: sedentary (G0), walk (G1), walk an extra activity (G2), and walk and 2 extra activities (G3). All participants performed the following assessments: questionnaire IPAQ, AAHPERD battery tests, blood pressure, lipid profile and body composition. Results: The results suggest that practice of non supervised walk (G1) promotes limited benefits to the health when compared to G3 (general functional fitness index $=273.4 \pm 111$ vs $340.6 \pm 92$; diastolic blood pressure $=80.0 \pm 8$ vs $75.4 \pm 7 \mathrm{mmHg}$; HDL cholesterol $=44.3 \pm 10$ vs $50.1 \pm 10 \mathrm{mg} / \mathrm{dL}$ respectively). Conclusions: The increased number of activities (G3) was considered beneficial to reduce the risk factors of cardiovascular disease compared to sedentary (G0) and walk (G1) groups.

Key-word: Physical exercise, aerobic capacity, fitness, health, aging. 


\section{Referências bibliográficas}

1. IBGE. Indicadores sócio-demográficos e de saúde no Brasil. Disponível em: www.ibge.gov.br; 2009 [cited 13/nov/2011 novembro].

2. WHO. Word Health Organization - Ageing. Disponível em: http://www.who.int/topics/ageing/en/; 2012 [cited 03/2012]

3. Matsudo MM, Matsudo VKR, Neto TLB. Impacto do envelhecimento nas variáveis antropométricas, neuromotoras e metabólicas da aptidão física. Rev Bras Ciênc Mov. 2000; 8:21-32.

4. Penha JC, Picarro Ida C, de Barros Neto TL. Evolution of physical fitness and functional capacity in active elderly women over 50 years of age according to chronological age in Santos city. Ciênc Saúde Coletiva. . 2012;17:245-53. Epub 2012/01/06. Evolucao da aptidao fisica e capacidade funcional de mulheres ativas acima de 50 anos de idade de acordo com a idade cronologica, na cidade de Santos.

5. Goncalves LH, Silva AH, Mazo GZ, Benedetti TR, dos Santos SM, Marques S, et al. Institutionalized elderly: functional capacity and physical fitness. Cad Saúde Pública. Ministerio da Saude, Fundacao Oswaldo Cruz, Escola Nacional de Saude Publica. 2010;26:1738-46. Epub 2010/09/30. O idoso institucionalizado: avaliacao da capacidade funcional e aptidao fisica.

6. Pauli JR, Souza LS, Zago AS, Gobbi S. Influência de 12 anos de prática de atividade física regular em programa supervisionado para idosos. Rev Bras de Cineantrop e Desemp Hum. 2009;11:255-60.

7. Balboa-Castillo T, Leon-Munoz LM, Graciani A, RodriguezArtalejo F, Guallar-Castillon P. Longitudinal association of physical activity and sedentary behavior during leisure time with health-related quality of life in community-dwelling older adults. Health Qual Life Outocomes.2011;9:47. Epub 2011/06/29.

8. Aidar FJ, de Oliveira RJ, Silva AJ, de Matos DG, Carneiro AL, Garrido N, et al. The influence of the level of physical activity and human development in the quality of life in survivors of stroke. Health Qual Life Outocomes.2011;9:89. Epub 2011/ $10 / 14$.

9. Hamer M, Stamatakis E. Physical activity and risk of cardiovascular disease events: inflammatory and metabolic mechanisms. Med Sci Sports Exerc. 2009;41:1206-11.

10. Kemmler W, Von Stengel S, Engelke K, Kalender WA. Exercise decreases the risk of metabolic syndrome in elderly females. Med Sci Sports Exerc. 2009;41:297-305.

11. Zago AS, Park JY, Fenty-Stewart N, Kokubun E, Brown MD. Effects of aerobic exercise on the blood pressure, oxidative stress and eNOS gene polymorphism in pre-hypertensive older people. Eur J Appl Physiol. 2010;110:825-32. Epub 2010/07/09.

12. Gerage AM, Forjaz CL, Nascimento MA, Januario RS, Polito MD, Cyrino ES. Cardiovascular adaptations to resistance training in elderly postmenopausal women. Int J Sports Med. 2013; Epub 2013/03/06.

13. Zaitune MP, Barros MB, Cesar CL, Carandina L, Goldbaum M. Variables associated with sedentary leisure time in the elderly in Campinas, Sao Paulo State, Brazil. Cad Saúde Pública. / Ministerio da Saude, Fundacao Oswaldo Cruz, Escola Nacional de Saude Publica. 2007;23:1329-38. Epub 2007/06/05. Fatores associados ao sedentarismo no lazer em idosos, Campinas, Sao Paulo, Brasil.

14. Fagard RH, Cornelissen VA. Effect of exercise on blood pres- sure control in hypertensive patients. Eur J Cardiovasc Prev Rehabil. 2007;14:12-7.

15. Chodzko-Zajko WJ, Proctor DN, Fiatarone Singh MA, Minson CT, Nigg CR, Salem GJ, et al. American College of Sports Medicine position stand. Exercise and physical activity for older adults. Med Sci Sports Exerc. 2009;41:1510-30.

16. Hallal PC, Andersen LB, Bull FC, Guthold R, Haskell W, Ekelund U. Global physical activity levels: surveillance progress, pitfalls, and prospects. Lancet. 2012;380(9838):247-57. Epub 2012/07/24.

17. Pescatello LS, Franklin BA, Fagard R, Farquhar WB, Kelley GA, Ray CA. American College of Sports Medicine position stand. Exercise and hypertension. Med Sci Sports Exerc. 2004;36:533-53.

18. Prajapati SK, Mansfield A, Gage WH, Brooks D, Mcllroy WE. Cardiovascular responses associated with daily walking in subacute stroke. Stroke research and treatment. 2013; 2013:612458. Epub 2013/03/12.

19. Hunter GR, Bickel CS, Del Corral P, Byrne NM, Hills AP, LarsonMeyer DE, et al. Age, muscle fatigue, and walking endurance in pre-menopausal women. Eur J Appl Physiol. 2011;111:71523. Epub 2010/10/26.

20. Murtagh EM, Murphy MH, Boone-Heinonen J. Walking: the first steps in cardiovascular disease prevention. Curr Opin Cardiol. 2010;25:490-6. Epub 2010/07/14.

21. Matsudo SMM, Matsudo VKR, Araújo TL. Perfil do nível de atividade física e capacidade funcional de mulheres maiores de 50 anos de idade de acordo com a idade cronológica. Rev Bras de Ativ Fis Saúde. 2001;6:12-24.

22. Zago AS, Gobbi S. Valores Normativos da aptidão funcional de mulheres de 60 a 70 anos. Rev Bras Ciênc Mov. 2003;11:7786.

23. Kline GM, Porcari JP, Hintermeister R, Freedson PS, Ward A, McCarron RF, et al. Estimation of VO2max from a one-mile track walk, gender, age, and body weight. Med Sci Sports Exerc. 1987;19:253-9.

24. Sociedade Brasileira de Hipertensão. VI Diretrizes Brasileiras de Hipertensão. Rev Bras Hipertens. 2010;13:1-66.

25. Friedewald WT, Levy RI, Fredrickson DS. Estimation of the concentration of low-density lipoprotein cholesterol in plasma, without use of the preparative ultracentrifuge. Clin Chem. 1972;18:499-502. Epub 1972/06/01.

26. Suzuki CS, Moraes SA, Freitas IC. Physical activity and correlates among adults living in Ribeirao Preto, Southeastern Brazil. Rev Saúde Pública. 2011;45:311-20. Epub 2011/03/18.

27. Caspersen CJ, Powell KE, Christenson GM. Physical activity, exercise, and physical fitness: definitions and distinctions for health-related research. Public Health Rep. 1985;100:126-31. Epub 1985/03/01.

28. Bailey DM, Davies B, Young IS, Jackson MJ, Davison GW, Isaacson R, et al. Epr spectroscopic evidence of free radical outflow from an isolated muscle bed in exercising humans: functional significance of decreasing intracellular $\mathrm{PO}_{2}$ vs. increasing $\mathrm{O}_{2}$ flux. Adv Exp Med Biol. 2003;540:297-303. Epub 2004/06/04.

29. Zago AS, Kokubun E, Fenty-Stewart N, Park JY, Attipoe S, Hagberg $\mathrm{J}$, et al. Effect of physical activity and t-786C polymorphism in blood pressure and blood flow in the elderly. Arq Bras Cardiol. 2010;95:510-6. 
30. Durstine JL, Grandjean PW, Davis PG, Ferguson MA, Alderson NL, DuBose KD. Blood lipid and lipoprotein adaptations to exercise: a quantitative analysis. Sports Med. 2001;31:1033-62. Epub 2001/12/12.

31. Sposito AC, Caramelli B, Fonseca FA, Bertolami MC, Afiune Neto A, Souza AD, et al. [IV Brazilian Guideline for Dyslipidemia and Atherosclerosis prevention: Department of Atherosclerosis of Brazilian Society of Cardiology]. Arq Bras Cardiol. 2007;88 Suppl 1:2-19. Epub 2007/05/23. IV Diretriz Brasileira sobre Dislipidemias e Prevencao da Aterosclerose: Departamento de Aterosclerose da Sociedade Brasileira de Cardiologia.

32. Guedes DP, Goncalves LA. Impact of the habitual physical activity on lipid profile in adults. Arq Bras Endocrinol Metab. 2007;51:72-8.

33. Marques E, Carvalho J, Soares JM, Marques F, Mota J. Effects of resistance and multicomponent exercise on lipid profiles of older women. Maturitas. 2009;63:84-8. Epub 2009/04/10.
34. Straznicky NE, Grima MT, Lambert EA, Eikelis N, Dawood T, Lambert GW, et al. Exercise augments weight loss induced improvement in renal function in obese metabolic syndrome individuals. J Hypertens. 2011;29:553-64. Epub 2010/12/02.

35. Mello D, Rosa G, Portela BO, Verdini MLP, Dantas EHM. Efeitos de um programa de caminhada sobre parâmetros biofísicos de mulheres com sobrepeso assistidas pelo Programa de Saúde da Família (PSF). Rev Bras Ativ Fís. Saúde. 2010;15:224-8.

36. Puglisi MJ, Vaishnav U, Shrestha S, Torres-Gonzalez M, Wood RJ, Volek JS, et al. Raisins and additional walking have distinct effects on plasma lipids and inflammatory cytokines. Lipids Health Dis. 2008;7:14. Epub 2008/04/18.

37. Tambalis K, Panagiotakos DB, Kavouras SA, Sidossis LS. Responses of blood lipids to aerobic, resistance, and combined aerobic with resistance exercise training: a systematic review of current evidence. Angiology. 2009;60:614-32. Epub 2008/11/01. 